\title{
A kinetic model of $T$ cell autoreactivity in autoimmune diseases
}

\author{
M.P. Machado $\operatorname{Ramos}^{a)}$, C. Ribeiro ${ }^{b)}$, A.J. Soares ${ }^{c}$ \\ Centro de Matemática, Universidade do Minho, Portugal \\ a) mpr@math.uminho.pt, ${ }^{b)}$ cribeiro@math.uminho.pt, ${ }^{c)}$ ajsoares@math.uminho.pt
}

(Revision, July 4, 2019)

\begin{abstract}
We construct a mathematical model of kinetic type in order to describe the immune system interactions in the context of autoimmune disease. The interacting populations are self-antigen presenting cells, self reactive $\mathrm{T}$ cells and the set of immunosuppressive cells consisting of regulatory $\mathrm{T}$ cells and Natural Killer cells. The main aim of our work is to develop a qualitative analysis of the model equations and investigate the existence of biologically realistic solutions. Having this goal in mind we describe the interactions between cells during an autoimmune reaction based on biological considerations that are given in the literature and we show that the corresponding system of integro-differential equations has finite positive solutions. The asymptotic behaviour of the solution of the system is also studied. We complement our mathematical analysis with numerical simulations that study the sensitivity of the model to parameters related to proliferation of immunosuppressive cells, destruction of self-antigen presenting cells and self reactive $\mathrm{T}$ cells and tolerance of SRTCs to self-antigens.
\end{abstract}

Keywords: Mathematical biology. Modelling. Kinetic theory. Autoimmune diseases. Cellular interactions. Non-linear ODE's.

AMS Subject Classification: 82C40, 92B05, 97M60, 34A34.

\section{Introduction}

The number of people affected by autoimmune diseases is on the rise, especially in Western countries [8]. This debilitating condition takes a devastating toll on affected families and has a considerable economic impact on both families and states. There are more than 80 different autoimmune diseases, such as inflammatory bowl disease, type 1 diabetes and multiple sclerosis. The exact cause of this surge remains unclear, but environmental changes associated with industrialization have been long suspected. Biologically, the mechanisms leading to autoimmune disease involve many factors and depend on a complex combination of genetic, epigenetic, molecular and cellular elements that result in pathogenic inflammatory responses in peripheral tissues commanded by $\mathrm{CD}^{+}{ }^{+}$effector memory $\mathrm{T}$ cells that react to self-antigens [13]. 
The role of certain cell populations, such as regulatory T lymphocytes (Tregs) and Natural Killer cells (NKCs), in controlling autoimmunity is demonstrated in a variety of experimental animal models. Also, there is a significant number of studies suggesting that defective regulation by Tregs or NKCs may be an underlying cause of human autoimmune diseases. The fact that such cell populations are an essential component of immune homoeostasis can potentially provide a therapeutic opportunity for active immune regulation that can have a long time effect in controlling the development of autoimmunity [9]. The importance of such cell populations in the mechanisms of suppression of autoimmunity has constituted the first motivation for the development of our mathematical model.

On the other hand, the application of mathematical models to biological and medical problems has stimulated a growing interest in the scientific community due to the recognized capability of such models in giving valuable predictions concerning the biological or medical problem. See, for example, references $[21,22,26]$ which collect several works on this topic

In general, such mathematical models are based on non-linear systems of differential equations with quadratic terms that describe the interactions between cells of the different populations. In particular, a kinetic modelling approach, like the one first developed in [6] for the tumour growth and then used in many other papers, see for example, $[2,3,4,7]$, is oriented to describe the interactions between the tumour and immune system that occur at a cellular level and thus can give some insights about the early stage of the disease. At the same time, such a model provides a macroscopic analogue that can be derived from the kinetic approach, which can be used either to describe successive biological stages in which macroscopic structures are more relevant or to investigate mathematically some qualitative properties of the solution and their counterpart in the biological reality. The scientific interest of the kinetic modelling approach constitutes the second motivation for our work.

In this article our principal goal is to construct a biologically realistic mathematical model describing autoimmunity, since, to the best of our knowledge, there is very few work on mathematical modelling of the process of autoimmunity in the literature $[12,18,28]$. This is certainly true in relation to mathematical models applying kinetic theory in describing the interactions between cell populations involved in the process $[12,18]$. If, on the one hand, building up a system of equations based on acceptable biological principles is in itself an important contribution to the field, on the other hand making sure that such a system is analytically well behaved is also fundamental when establishing a consistent mathematical model. In fact, constructing a mathematical model of integro-differential equations in which the biological premisses are compatible with certain mathematical requirements on the model equations, such as existence and uniqueness of solution of the system equations, positivity of solution and non appearance of blowups, can be a difficult task to undergo.

We believe that the main contribution of this work is that we were able to base the model equations describing the interaction of cells during an autoimmune reaction on biological considerations that are given in the literature, and are explained in Section 2, but also we were able to show that the corresponding system of integro-differential equations has finite positive solutions and predict the asymptotic behaviour of these solutions.

More in detail, our model describes the interactions among three different population cells, namely self antigen presenting cells (SAPCs), self reactive T cells (SRTCs) and immunosupressive cells (ISCs), such as Tregs and NKCs. The cells of the interacting populations are 
characterized by a microscopic functional state or activity variable, $u \in[0,1]$, which defines a specific biological function of each cell. The behaviour of each interacting population is described, at the kinetic (cellular) level, by its distribution function. The model equations then constitute a nonlinear system of integro-differential type that describes the evolution of the distribution functions.

As expected, it is important to guarantee that the mathematical model describes as realistically as possible the evolution of the cellular system being considered and that the parameters characterizing the model can be effectively related to biophysical quantities. Therefore, from the biological theory we should derive certain conditions that must be verified by the parameters of our model. Having this idea in mind, we present, in Section 2, the biological theory on autoimmunity that we take into consideration when establishing our mathematical model. In Section 3, we first describe, mathematically, the microscopic cellular interactions among the populations considered in our analysis and then we derive the model equations in its kinetic formulation as well as in the corresponding macroscopic setting. The qualitative analysis of the model equations is performed in Section 4, where we prove the existence of a global solution to the macroscopic system and investigate its asymptotic behaviour. In Section 5, we apply our kinetic system to study the regulatory effect of ISCs on the proliferation and activation of SRTCs in an autoimmune reaction. The system is solved numerically by discretizing the kinetic equations in the activation state variable and using a quadrature rule in the numerical integration. Finally, in Section 6, we summarize our results and discuss some possible ideas to be developed as a continuation of our paper.

\section{Biological considerations}

In this section we give an overview of how autoimmunity plays out in individuals with a predisposition for the disease and how, so called regulatory cells combat the autoimmune process. We base this overview on several references, $[1,8,9,11,13,16,19,20,23,24,25,27$, 29 , that can be found in the literature on this subject and where the mechanisms involved in autoimmunity are described.

It is well known that $\mathrm{T}$ cells derive from the common lymphoid in the bone marrow and migrate through the bloodstream into the thymus, where they undergo several different maturation phases. An important role of thymic maturation of $\mathrm{T}$ cells is the positive selection for those $\mathrm{T}$ cells that recognize self-MHC molecules and negative selection against those $\mathrm{T}$ cells that react to self-antigens, in other words that have antigen receptors specific to self-antigens. The development of autoimmune disease is related to the breakdown of selftolerance mechanisms, that in a healthy individual prevent the majority of self reactive $\mathrm{T}$ cell clones from entering the periphery. That being said, even in a healthy human body there exist circulating SRTCs [11]. SRTCs are usually controlled by both intrinsic and extrinsic mechanisms of tolerance in the periphery.

The intrinsic mechanism involves a $\mathrm{T}$ cell receiving a tolerising TCR signal in advance of encountering its antigen, presented by a professional antigen presenting cell (APC), so that the $\mathrm{T}$ cell will not become fully activated. The extrinsic tolerance mechanism is the dominant form of immune suppression carried out by regulatory cell populations such as Tregs and NKCs. In people with genetic predisposition to autoimmunity, one or several of these tolerance mechanisms are defective, resulting in expansion of SRTCs that cannot 
be controlled by, for example Tregs. The expansion of SRTCs is due to their exposure to self-antigens by SAPCs.

In immunology, an adapted immune response occurs when immature dentritic cells (DCs), which are the most important APCs, settle at the site of infection, become activated and undergo maturation. Meanwhile, naive T cells, each having a specific antigen receptor, constantly circulate through the peripheral lymphoid tissues, browsing many DCs as they carry out brief contacts, and receiving two signals, one allows the discrimination of the antigen presented by DCs and the other allows the interplay with co-stimulatory molecules on the same DCs. After making a stable interaction with DCs presenting their cognate antigen, naive $\mathrm{T}$ cells can be activated and proliferate into memory effector $\mathrm{T}$ cells. The proliferation of $\mathrm{T}$ cells is considerable and is driven by cytokine interleukin- 2 (IL-2), which can be produced by DCs. In fact, T cells undergo numerous rounds of cell division, following exposure to an intracellular pathogen, expanding up to 50.000 fold in the course of about a week [16]. The same process of activation/proliferation takes place in an autoimmune response to self antigens, but in this case SRTCs have antigen receptors specific to self-antigens. The cloned SRTCs migrate to their targeted tissue where cytotoxic mechanisms and uncontrolled inflammation induced by cytokines produced by these $\mathrm{T}$ cells result in tissue damage, consequently causing autoimmune disease. The damage to host tissue results in an increased concentration of self-antigens that will activate more SRTCs when presented to these cells by SAPCs, eventually worsening the autoimmune attack to host tissues.

Regulatory $\mathrm{T}$ cells are a subpopulation of $\mathrm{CD} 4^{+} \mathrm{T}$ cells believed to be important mediators within the immune system. Actually, the importance of regulatory $\mathrm{T}$ lymphocytes in the control of autoimmunity is scientifically a well established theory [1, 9, 20, 24, 25, 29], in fact, there are several studies that suggest that Treg deficits may be one of the causes of human autoimmune disease. An important line of research on autoimmunity is to study the mechanisms of suppression performed by Tregs, which to date are not fully understood. It is believed that Tregs can carry out their suppressive function either through contact dependent mechanisms, such as directly targeting the action of effector $\mathrm{T}$ cells as well as modulating the maturation and/or function of APCs which are required for the activation of the former, and/or through contact-independent mechanisms [20]. Here we are interested in the contact dependent mechanisms induced by the interaction between Tregs, SRTCs and SAPCs. Tregs down-modulate the capacity of APCs to initiate immune responses, and therefore preventing the ability of APCs to activate effector T cells. Another potential mechanism of Treg mediated suppression is cell-contact dependent cytolysis of target cells. It has been shown that activated Tregs by APCs induce apoptosis of effector T cells through a tumor-necrosis factor related apoptosis-inducing ligand death receptor pathway. Therefore, in autoimmunity, the main functional properties of Tregs involve suppression by modulation of SAPC maturation or function and suppression by direct or indirect killing of SRTCs and SAPCs.

Increasing evidence shows that Natural Killer cells play a relevant role in organic-specific and systemic autoimmune diseases [23, 27]. As several other cells belonging to the innate arm of the immune system, NKCs can secrete cytokines and chemokines. Both activation of cytolytic response and secretion of regulating soluble factors are dependent on a wide variety of surface and intracellular receptors that, interact with the appropriate ligand leading to activation or inhibition of a given cell function. As is usual in a biological system, the balance between opposite signals is responsible for the outcome in the microenviroment, and therefore 
NKCs can both influence and regulate the activities of adaptive immune responses, in particular of effector T cells and APCs through well identified surface receptors. It appears that NKCs may play opposite roles with both regulatory and inducer activity in autoimmunity. Of course, here we are interested in their regulatory activity. There is clear evidence that NKCs can recognize self-antigens which express ligands for activating receptors and therefore it is believed that NKCs can aggress both SRTCs and SAPCs upon triggering with toll-like receptor (TLR) or stimulation with IL2 or IL15 cytokines. There are consistent findings that indicate that NKCs can strongly regulate $\mathrm{T}$ cell responses and influence adaptive immunity. It is known that NKCs can interact with APCs that produce interleukin 12 (also known as NKC stimulating factor) which triggers both proliferation and cytolytic activity of NKCs. Several reports have shown that IL2-activated NKCs can lyse SAPCs and that NKC-SAPC interaction can lead to cytokine production. Indeed, the NKC mediated elimination of a given SAPC before self-antigen presentation to SRTCs should impede an optimal T cell activation, and therefore the adaptive immune response can be shut down by NKCs. In conclusion, NKCs possess the ability of protecting from the occurrence of autoimmune disease through secretion of immune regulating cytokines, such as IL10, IL5 and IL13 and by eliminating SAPCs and SRTCs through, for example, the triggering of activating receptors.

Autoimmunity diseases are, in many cases, chronic, meaning that patients suffering from theses conditions go through relapses, where symptoms return after a certain period of remittance (where symptoms are absent). In several autoimmune diseases this relapse-remittance behaviour occurs even in the absence of treatment. Here, we do not model the relapseremittance behaviour. Our model concentrates on the cellular dynamics when an autoimmune episode occurs, particularly on the interplay between SAPCs-SRTCs-Tregs/NKCs that can either result in an autoimmune cascade and consequently in illness or in an aborted autoimmune response by regulation via Tregs /NKCs.

In what follows, we model the interactions between SAPCs and SRTCs considering that the activation/proliferation of SRTCs is due to their exposure to self-antigens by SAPCs. We also consider that the damage to host tissue, resulting from inflammation induced by cytokines produced by SRTCs, increases the number of SAPCs that will activate more SRTCs. Regulation of the autoimmune process is modelled through the interactions between SAPCs and ISCs and between SRTCs and ISCs. We consider that ISCs, on the one hand downgrade the function of SAPCs aand SRTCs, and on the other hand eliminate both SAPCs and SRTCs.

\section{The mathematical model for autoimmunity}

In this section, we construct a mathematical model based on the Boltzmann-like kinetic theory [10] for the autoimmune response against self antigens. We start with the description of the binary cellular interactions and then we derive the kinetic equations giving the evolution of the distribution functions associated to the considered populations. Finally we define macroscopic variables in terms of suitable mean quantities of the distribution functions and formally derive the macroscopic balance equations for these variables.

Our approach follows the research line first proposed by Bellomo and Forni in [6] and then essentially developed in papers $[2,3,4,7]$. For a further reading on the interplay between 
mathematics and biology, we suggest the recent review on the subject presented in [5] and the references therein cited.

\subsection{Microscopic cellular interactions}

We consider a biological system of three interacting cell populations $p_{i}, i=1,2,3$, that are involved in the development of autoimmunity. More precisely, we consider the population $p_{1}$ of self antigen presenting cells, the population $p_{2}$ of self reactive $\mathrm{T}$ cells, and the population $p_{3}$ of immunosuppressive cells mainly constituted by $\mathrm{T}$ regulating cells and Natural Killer $\mathrm{T}$ cells.

\section{The cellular activity}

Following the biological considerations of Section 2, the functional state of each cell population $p_{i}$ is described by a real variable $u \in[0,1]$, called the biological activity, which is defined as follows.

- For the population $p_{1}$ of SAPCs, the activity variable $u$ represents the ability to stimulate and activate the SRTCs. If $u$ is equal to zero, then the stimulation by SAPCs does not activate the SRTCs and, therefore, does not induce an autoimmune response. Increasing values of $u$ indicate a growing efficiency of SAPCs in activating the SRTCs.

- For the population $p_{2}$ of SRTCs, the activity variable $u$ is the quantity of cytokines secreted by SRTCs. If $u$ is equal to zero, then SRTCs do not produce cytokines, corresponding to the case where SRTCs are tolerant to self-antigens, therefore recognizing them as non offensive agents. Increasing values of $u$ indicate a growing efficiency of SRTCs in producing cytokines that will trigger an inflammatory cascade and, consequently, an autoimmune response.

- For the population $p_{3}$ of ISCs, the activity variable $u$ is the ability to inhibit the autoimmune response by either suppressing the activity of SAPCs and SRTCs, or eliminating SAPCs and SRTCs. We will, however, for this population neglect the presence of internal degrees of freedom.

The internal state of the biological system is described by the set of distribution functions associated to the considered populations, $f_{i}:[0, \infty] \times[0,1] \rightarrow \mathbb{R}^{+}, i=1,2,3$, such that $f_{i}(t, u)$ gives the number of cells of population $p_{i}$ with activity $u \in[0,1]$ at time $t \geq 0$. The expected number of cells of each population $p_{i}$ at time $t \geq 0$ is then given by

$$
n_{i}(t)=\int_{0}^{1} f_{i}(t, u) \mathrm{d} u, \quad i=1,2,3 .
$$

\section{Our assumptions}

In our mathematical model, as a simplification of the biological reality, we consider the following assumptions.

$\left(A_{1}\right)$ We only consider binary interactions between cells.

$\left(\mathrm{A}_{2}\right)$ The interactions between cells are instantaneous and homogeneous in space. 
$\left(\mathrm{A}_{3}\right)$ The binary interactions between cells of populations $p_{1}$ (SAPCs), $p_{2}$ (SRTCs) and $p_{3}$ (ISCs) can change the activation of SAPCs and SRTCs as well as the size of each population by both creating more SAPCs, SRTCs and ISCs or by destroying SAPC and SRTCs. In the first case, the interactions are conservative, whereas in the second case they are either proliferative or destructive interactions.

$\left(\mathrm{A}_{4}\right)$ For population $p_{3}$, the distribution function is independent of its functional state, so that $f_{3}=f_{3}(t)$.

Assumption $\left(A_{1}\right)$ is rather natural when describing biological cellular systems and means that all effective interactions in our model occur between a pair of cells. Assumption $\left(\mathrm{A}_{2}\right)$ indicates that our model does not describe the relapse-remittance behaviour of autoimmune disease (see Section 2) and that the evolution of our biological system is uniform with respect to space variables. Assumption $\left(\mathrm{A}_{3}\right)$ is motivated by the biological considerations presented in Section 2, in particular by the fact that during an immune response to either antigens (in immunity) or self-antigens (in autoimmunity) there occurs proliferation of both effector $\mathrm{T}$ cells and regulatory $\mathrm{T}$ cells as well as an increase of circulating APCs. Also, the job of ISCs during such an immune reaction is to down regulate both proliferation of effector $\mathrm{T}$ cells and APCs and their activity. Finally, as a simplification we assume in $\left(\mathrm{A}_{4}\right)$ that the population of immunosuppressive cells is homogeneous with respect to their biological activity, since in our model we do not consider the effect of the interactions between the cell populations on the activity of Tregs and NKCs. Therefore, we neglect the presence of internal degrees of freedom for this population.

\section{The microscopic interactions}

In order to describe the evolution of the distribution functions $f_{i}$, it is necessary to model the microscopic binary interactions between SAPCs, SRTCs and ISCs, in accordance with the biological considerations described in Section 2 and with the assumptions listed above.

\section{SAPCs conservative interactions}

We consider conservative interactions between SAPCs and SRTCs as well as between SAPCs and ISCs, which do not modify the total number of SAPCs. The interactions between SAPCs and SRTCs affect the activity of SAPCs by increasing their capacity of activating SRTCs, which means increasing the functional state of SAPCs. This in turn implies that SRTCs will better recognize self-antigens transported by SAPCs as foreign agents and that a more efficient stimulation of SRTCs by contact with SAPCs will be obtained.

On the other hand, conservative interactions between SAPCs and ISCs result in a decreasing ability of SAPCs to present and activate SRTCs. Biologically, this means that ISCs induce a suppressive effect in the function of SAPCs.

The gain and loss terms corresponding to these conservative interactions are given by

$$
\begin{gathered}
C_{1}[f](t, u)=\sum_{j=2,3} \int_{0}^{1} \int_{0}^{1} \eta_{1 j}^{(c)}(v, w) \psi_{1 j}^{(1)}(v, w ; u) f_{1}(t, v) f_{j}(t, w) \mathrm{d} v \mathrm{~d} w \\
-f_{1}(t, u) \sum_{j=2,3} \int_{0}^{1} \eta_{1 j}^{(c)}(u, w) f_{j}(t, w) \mathrm{d} w
\end{gathered}
$$


where the encounter rates $\eta_{12}^{(c)}$ and $\eta_{13}^{(c)}$ are

$$
\eta_{12}^{(c)}(v, w)=c_{12}(v-1)^{2}, \quad \eta_{13}^{(c)}(v, w)=c_{13} v^{2}, \quad \text { for } \quad v, w \in[0,1]
$$

and $c_{12}$ and $c_{13}$ are positive constants characterizing the progress of SAPCs towards increasing $\left(c_{12}\right)$ or decreasing $\left(c_{13}\right)$ their activation state due to the interaction with SRTCs and ISCs, respectively. Moreover, the corresponding transition probability densities are given by the following nonnegative functions

$$
\psi_{12}^{(1)}(v, w ; u)=\left\{\begin{array}{cl}
\frac{2(u-v)}{(v-1)^{2}} & \text { for } u>v \\
0 & \text { for } u \leq v
\end{array}, \quad \psi_{13}^{(1)}(v, w ; u)=\left\{\begin{array}{cl}
\frac{2(v-u)}{v^{2}} & \text { for } u<v \\
0 & \text { for } u \geq v
\end{array}\right.\right.
$$

which satisfy the conditions

$$
\int_{0}^{1} \psi_{1 j}^{(1)}(v, w ; u) d u=1, \quad j=2,3
$$

\section{SRTCs conservative interactions}

The conservative interactions between SRTCs and SAPCs increase the production of cytokines by SRTCs. This has to do with the fact that SRTCs are effector memory $\mathrm{T}$ cells that are able to retain information. On the other hand, SRTCs participate in conservative interactions with ISCs, that contribute to weaken the production of cytokines by SRTCs, due to the inhibiting effect of ISCs on the SRTC function.

Accordingly, we define the corresponding gain and loss terms as follows

$$
\begin{gathered}
C_{2}[f](t, u)=\sum_{j=1,3} \int_{0}^{1} \int_{0}^{1} \eta_{2 j}^{(c)}(v, w) \psi_{2 j}^{(2)}(v, w ; u) f_{2}(t, v) f_{j}(t, w) \mathrm{d} v \mathrm{~d} w \\
-f_{2}(t, u) \sum_{j=1,3} \int_{0}^{1} \eta_{2 j}^{(c)}(u, w) f_{j}(t, w) \mathrm{d} w .
\end{gathered}
$$

The respective encounter rates are given by

$$
\eta_{21}^{(c)}(v, w)=c_{21}(v-1)^{2}, \quad \eta_{23}^{(c)}(v, w)=c_{23} v^{2}, \quad \text { for } \quad v, w \in[0,1],
$$

where $v, w \in[0,1]$ and $c_{21}$ and $c_{23}$ are non-negative constants.

The transition probability densities $\psi_{21}^{(2)}$ and $\psi_{23}^{(2)}$ for these interactions are defined as

$$
\begin{aligned}
& \psi_{21}^{(2)}(v, w ; u)=\left\{\begin{array}{cc}
\frac{2(u-v)}{(v-1)^{2}} & \text { for } u>v, \quad w^{*}<w \leq 1 \\
0 & \text { for } u \leq v \text { or } 0 \leq w \leq w^{*}
\end{array}\right. \\
& \psi_{23}^{(2)}(v, w ; u)=\left\{\begin{array}{cc}
\frac{2(v-u)}{v^{2}} & \text { for } u<v \\
0 & \text { for } u \geq v
\end{array}\right.
\end{aligned}
$$


and they satisfy a condition analogous to (2). Furthermore, $\left.w^{*} \in\right] 0,1[$ is a parameter of the model, that is related to the tolerance of SRTCs to self-antigens, in the sense that the greater the value of $w^{*}$ the less efficient are SAPCs in increasing the activity of SRTCs after encounter.

\section{SRTCs proliferative interactions}

We consider that proliferative encounters between SRTCs and SAPCs increase the number of SRTCs after presentation of self-antigens by SAPCs. The gain term is given by

$$
P_{2}[f](t, u)=\int_{0}^{1} \int_{0}^{1} P_{21}^{(2)}(v, w ; u) f_{2}(t, v) f_{1}(t, w) \mathrm{d} v \mathrm{~d} w,
$$

where the proliferation transition rate $P_{21}^{(2)}(v, w ; u)$ is

$$
P_{21}^{(2)}(v, w ; u)=p_{21}^{(2)}(u, w) \delta(v-u),
$$

with $p_{21}^{(2)}(u, w)$ being the proliferation rate and $\delta$ the Dirac delta function. This means that the newborn cells inherit the same aggressive state as the mother cell. Therefore, the gain term simplifies to, see [7],

$$
P_{2}[f](t, u)=f_{2}(t, u) \int_{0}^{1} p_{21}^{(2)}(u, w) f_{1}(t, w) \mathrm{d} w .
$$

Here, we will assume that the proliferation rate $p_{21}^{(2)}(u, w)$ is a non-negative constant,

$$
p_{21}^{(2)}(u, w)=p_{21}, \quad u, w \in[0,1] .
$$

\section{SAPCs proliferative interactions}

The autoimmune cascade, resulting from the cytokine production by SRTCs after encounter with self-antigens, causes a damage to the host tissue which, in turn, increases the concentration of self-antigens that will be transported by SAPCs. We then consider that encounters between SAPCs and SRTCs increase the number of SAPCs and the corresponding gain term is given by

$$
P_{1}[f](t, u)=f_{1}(t, u) \int_{0}^{1} p_{12}^{(1)}(u, w) f_{2}(t, w) \mathrm{d} w .
$$

We will consider that the new SAPCs resulting from these interactions have the same aggressive state as those of the mother SAPCs, and assume that the proliferation rate $p_{12}^{(1)}(u, w)$ is a non-negative constant,

$$
p_{12}^{(1)}(u, w)=p_{12}, \quad u, w \in[0,1]
$$

ISCs proliferative interactions

Like other effector $\mathrm{T}$ cells, when participating in an autoimmune response, the ISCs require the activation by self-antigens on SAPCs and the possible costimulation result in the proliferation of Tregs and NKCs. This type of interactions is then of proliferative type for the ISCs and the corresponding gain term is given by

$$
P_{3}[f](t, u)=f_{3}(t) \int_{0}^{1} p_{31}^{(3)}(u, w) f_{1}(t, w) \mathrm{d} w .
$$


Once again, we consider that the newborn ISCs inherit the same aggressive state as the mother ISCs and that the proliferation rate $p_{31}^{(3)}(u, w)$ is a non-negative constant, that is

$$
p_{31}^{(3)}(u, w)=p_{31}, \quad u, w \in[0,1] .
$$

\section{SAPCs destructive interactions}

We consider that some interactions between SAPCs and ISCs result in the elimination of SAPCs and, thus, are of destructive type for SAPCs. The corresponding loss term is given by

$$
D_{1}[f](t, u)=f_{1}(t, u) \int_{0}^{1} d_{13}^{(1)}(u, w) f_{3}(t, w) \mathrm{d} w
$$

where the destruction rate $d_{13}^{(1)}(u, w)$ is assumed to be a non-negative constant,

$$
d_{13}^{(1)}(u, w)=d_{13}, \quad u, w \in[0,1] .
$$

SRTCs destructive interactions

Finally, we assume destructive encounters between SRTCs and ISCs that decrease the number of SRTCs by direct elimination of these last cells. The corresponding loss term is

$$
D_{2}[f](t, u)=f_{2}(t, u) \int_{0}^{1} d_{23}^{(1)}(u, w) f_{3}(t, w) \mathrm{d} w,
$$

where the destruction rate $d_{23}^{(1)}(u, w)$ is assumed to be a non-negative constant, that is

$$
d_{23}^{(1)}(u, w)=d_{23}, \quad u, w \in[0,1] .
$$

The description given here of the relevant interactions involved in our biological system is a crucial step in the derivation of the model equations giving the dynamics of the cellular populations in autoimmune diseases. Obtaining these model equations will be the objective of the next subsection.

\section{$3.2 \quad$ The model equations}

Starting from the description of the microscopic cellular dynamics given in the previous subsection, we will derive the evolution equations for the distribution functions $f_{i}(t, u), i=$ $1,2,3$, and then obtain the balance equations for the number of cells of each population defined by the $u$-averaged functions $n_{i}(t), i=1,2,3$.

\section{The kinetic evolution equations}

The evolution equations for the distribution functions $f_{i}, i=1,2,3$, can be derived as suitable balance equations which equate the time derivative of $f_{i}$ to the global interaction operator obtained by summing the respective individual operators of conservative, proliferative or destructive type, see [7]. The system so obtained consists of the following three coupled integro-differential equations

$$
\partial_{t} f_{1}(t, u)=2 c_{12} \int_{0}^{u}(u-v) f_{1}(t, v) \mathrm{d} v \int_{0}^{1} f_{2}(t, w) \mathrm{d} w-c_{12}(u-1)^{2} f_{1}(t, u) \int_{0}^{1} f_{2}(t, w) \mathrm{d} w
$$




$$
\begin{gathered}
+2 c_{13} f_{3}(t) \int_{u}^{1}(v-u) f_{1}(t, v) \mathrm{d} v-c_{13} u^{2} f_{1}(t, u) f_{3}(t) \\
+p_{12} f_{1}(t, u) \int_{0}^{1} f_{2}(t, w) \mathrm{d} w-d_{13} f_{1}(t, u) f_{3}(t) \\
\partial_{t} f_{2}(t, u)=\quad 2 c_{21} \int_{0}^{u}(u-v) f_{2}(t, v) d v \int_{w^{\star}}^{1} f_{1}(t, w) d w-c_{21}(u-1)^{2} \\
\quad f_{2}(t, u) \int_{0}^{1} f_{1}(t, w) d w+2 c_{23} f_{3}(t) \int_{u}^{1}(v-u) f_{2}(t, v) d v-c_{23} u^{2} f_{2}(t, u) f_{3}(t) \\
\quad+p_{21} f_{2}(t, u) \int_{0}^{1} f_{1}(t, w) d w-d_{23} f_{2}(t, u) f_{3}(t) \\
\dot{f}_{3}(t)=p_{31} f_{3}(t) \int_{0}^{1} f_{1}(t, w) d w
\end{gathered}
$$

for all $u \in[0,1]$ and $t \in \mathbb{R}^{+}$. In the above equations (5)-(7), we use the notation $p_{12}, p_{21}$, $p_{31}, d_{13}, d_{23}$ and $c_{13}, c_{21}, c_{23}$ that has been introduced in Subsection 3.1 for the proliferative, destructive and conservative rates, respectively.

Additionally, we write the initial conditions for $t=0$ of the system (5)-(7) as

$$
f_{1}(0, u)=f_{1}^{0}(u), \quad f_{2}(0, u)=f_{2}^{0}(u), \quad f_{3}(0)=f_{3}^{0} .
$$

\section{The macroscopic balance equations}

From the kinetic system (5)-(7), we can derive the corresponding macroscopic model describing the time evolution of the number of cells of each population, namely $n_{i}(t), i=1,2,3$, which have been defined in Section 3, see expression (1). The balance equations for the evolution of $n_{i}(t), i=1,2,3$, are obtained by suitable integration of the kinetic equations (5)-(7) over the biological activity variable $u \in[0,1]$. Conservative interactions do not give any contribution to the equations of $n_{i}(t)$, since they do not modify the number of cells of each population. Therefore, the system of ordinary differential equations obtained in this way is

$$
\begin{aligned}
& \dot{n}_{1}(t)=p_{12} n_{1}(t) n_{2}(t)-d_{13} n_{1}(t) n_{3}(t), \\
& \dot{n}_{2}(t)=p_{21} n_{2}(t) n_{1}(t)-d_{23} n_{2}(t) n_{3}(t), \\
& \dot{n}_{3}(t)=p_{31} n_{3}(t) n_{1}(t) .
\end{aligned}
$$

For this system, we consider the following initial data

$$
n_{1}(0)=n_{1}^{0}, \quad n_{2}(0)=n_{2}^{0}, \quad n_{3}(0)=n_{3}^{0}, \quad \text { with } \quad n_{i}^{0}>0 \quad \text { for } \quad i=1,2,3 .
$$

\section{Some notes on the model equations}

The system (9)-(11) of ordinary differential equations represents the macroscopic analogue of the kinetic system (5)-(7) of partial differential equations describing the microscopic dynamics between cells. 
As expected, the description obtained with the kinetic system is more detailed and reflects how the microscopic interactions among cells affect the activity of the various populations and how they contribute to the evolution of the distribution functions $f_{i}(t, u), i=1,2,3$. This system will be the one used in the numerical simulations performed in Section 5 .

The mathematical analysis of the kinetic system is obviously a complex task but the existence of a unique local solution to our initial value problem (5)-(7) and (8) can be stated. In fact, papers [2] and [3] prove local existence theorems for an initial value problem that is more general than the one formulated here. Therefore, such theorems guarantee the wellposedness of our initial value problem.

On the other hand, the description obtained with the corresponding macroscopic system (9)-(11) gives information at a global (macroscopic) scale and only reflects information about the changes on the number of cells of each population. Other aspects, in particular those associated to the cellular activity, are embedded in the macroscopic dynamics and are not directly recognizable in the balance equations.

The mathematical analysis of the macroscopic system is easier than that of the kinetic system and there are certain circumstances in which relevant information on the solution to the kinetic system can be extracted from the mathematical analysis of the macroscopic equations. This aspect will be exploited in Section 4. In fact, in our kinetic system (5)(7), we have considered that during proliferative encounters cloned cells inherit the same aggressive state as their mother cell, at a constant proliferation rate, and, additionally, that the destructive encounters occur at a constant destruction rate. Under these assumptions, it is proven in [4] that the boundedness of the solution of their macroscopic system implies the boundedness of the solution to their kinetic system. A careful analysis of the results stated in the above quoted paper [4] guarantees their application to our macroscopic and kinetic systems. Therefore, the boundedness of the solution to our macroscopic system (9)-(11), say $n_{i}(t), i=1,2,3$, also implies the boundedness of the solution to our kinetic system (5)(7), say $f_{i}(t, u), i=1,2,3$. More precisely, from paper [4], we can bound $\left\|f_{i}(t, \cdot)\right\|_{1}$, where $f_{i}(t, \cdot) \in L_{1}(0,1)$ and $\left\|f_{i}(t, \cdot)\right\|_{1}$ is the norm of $f_{i}(t, \cdot)$ in the Lebesgue space $L_{1}(0,1)$.

In paper [4], it is also proven that, under the same assumptions, if $n_{i}(t)$ blows up then so does $\left\|f_{i}(t, \cdot)\right\|_{1}$.

\section{Qualitative analysis of the model}

From the discussion developed at the end of the previous section, we can conclude that the basic information on the microscopic model given by the system (5)-(7) is contained in the corresponding macroscopic model (9)-(11). Therefore, in what follows we give a detailed qualitative description of the solutions to system (9)-(11), by introducing an additional assumption on the proliferative parameters $p_{21}$ and $p_{31}$. More specifically, we will prove in Theorem 4.1 the existence and uniqueness of a global solution to the Cauchy problem associated to our macroscopic system (9)-(11) with initial conditions (12) and study the asymptotic behaviour of the solution in the particular case where $p_{21} / p_{31}<1$.

We start with the following result concerning the positivity of the existing solutions to the Cauchy problem (9)-(11), (12). 
Lemma 4.1. Let $\underline{n}(t)=\left(n_{1}(t), n_{2}(t), n_{3}(t)\right)$ be a solution of the Cauchy problem (9)-(11), (12) defined on the time interval $[0, T]$, with $T$ such that $0<T<\infty$. Then $n_{1}(t)>0, n_{2}(t)>0$ and $n_{3}(t)>0$, for all $t \in[0, T]$.

Proof.

(i) First we prove the positivity of $n_{1}(t)$.

Let us assume that there exists $\left.\left.\bar{t}_{1} \in\right] 0, T\right]$ such that $n_{1}\left(\bar{t}_{1}\right)=0$. Then, we take $\bar{n}_{2}=n_{2}\left(\bar{t}_{1}\right)$ and $\bar{n}_{3}=n_{3}\left(\bar{t}_{1}\right)$ and consider the function $\underline{N}(t)=\left(N_{1}(t), N_{2}(t), N_{3}(t)\right)$ to be a solution to the differential system (9)-(11) with initial conditions

$$
N_{1}(0)=0, \quad N_{2}(0)=\bar{n}_{2} e^{d_{23} \bar{n}_{3} \bar{t}_{1}}, \quad N_{3}(0)=\bar{n}_{3} .
$$

Therefore, by the Picard method [14], we can easily obtain

$$
N_{1}(t)=0, \quad N_{3}(t)=\bar{n}_{3}, \quad \text { for } \quad t \in[0, T],
$$

and from the system (9)-(11), we have

$$
\dot{N}_{1}(t)=0, \quad \dot{N}_{2}(t)=-d_{23} \bar{n}_{3} N_{2}(t), \quad \dot{N}_{3}(t)=0,
$$

so that,

$$
N_{1}(t)=0, \quad N_{2}(t)=\bar{n}_{2} e^{d_{23} \bar{n}_{3}\left(\bar{t}_{1}-t\right)}, \quad N_{3}(t)=\bar{n}_{3}, \quad \text { for } \quad t \in[0, T] .
$$

In particular, for $t=\bar{t}_{1}$, we have

$$
N_{1}\left(\bar{t}_{1}\right)=0=n_{1}\left(\bar{t}_{1}\right), \quad N_{2}\left(\bar{t}_{1}\right)=\bar{n}_{2}=n_{2}\left(\bar{t}_{1}\right), \quad N_{3}\left(\bar{t}_{1}\right)=\bar{n}_{3}=n_{3}\left(\bar{t}_{1}\right) .
$$

Both solutions $\underline{n}(t)=\left(n_{1}(t), n_{2}(t), n_{3}(t)\right)$ and $\underline{N}(t)=\left(N_{1}(t), N_{2}(t), N_{3}(t)\right)$ to the system (9)(11) are defined on $[0, T]$ and take the same value at $t=\bar{t}_{1}$. Consequently, they coincide on the interval $[0, T]$. Then, we obtain

$$
n_{1}(0)=N_{1}(0)=0
$$

which contradicts the fact that $n_{1}(0)>0$. Therefore, there does not exist $\left.\left.\bar{t}_{1} \in\right] 0, T\right]$ such that $n_{1}\left(\bar{t}_{1}\right)=0$. Since the function $n_{1}(t)$ is continuous and $n_{1}(0)>0$, we conclude that $n_{1}(t)>0$ for all $t \in[0, T]$.

(ii) We now proceed to prove that $n_{2}(t)>0$ for all $t \in[0, T]$.

Analogously to what we have done in (i), let us assume that there exists $\left.\bar{t}_{2} \in\right] 0, T$ ] such that $n_{2}\left(\bar{t}_{2}\right)=0$. We put $\widetilde{n}_{1}=n_{1}\left(\bar{t}_{2}\right)$ and $\widetilde{n}_{3}=n_{3}\left(\bar{t}_{2}\right)$ and consider the function $\underline{N}(t)=$ $\left(N_{1}(t), N_{2}(t), N_{3}(t)\right)$ to be a solution to the system (9)-(11) satisfying the initial conditions

$$
N_{1}(0)=\widetilde{n}_{1} e^{d_{13} \int_{0}^{\bar{t}_{2}} N_{3}\left(t^{\prime}\right) d t^{\prime}}, \quad N_{2}(0)=0, \quad N_{3}(0)=\widetilde{n}_{3} e^{-p_{31} \int_{0}^{\bar{t}_{2}} N_{1}\left(t^{\prime}\right) d t^{\prime}} .
$$

By the Picard method [14], we then obtain

$$
N_{2}(t)=0, \quad t \in[0, T],
$$

and the system (9)-(11) gives

$$
\left.\left.\dot{N}_{1}(t)=-d_{13} N_{1}(t) N_{3}(t), \quad \dot{N}_{3}(t)=p_{31} N_{1}(t) N_{3}(t), \quad \text { for } \quad t \in\right] 0, T\right] .
$$


Consequently, we have

$$
\begin{aligned}
& N_{1}(t)=\widetilde{n}_{1} e^{d_{13}\left(\int_{0}^{\bar{t}_{2}} N_{3}\left(t^{\prime}\right) d t^{\prime}-\int_{0}^{t} N_{3}\left(t^{\prime}\right) d t^{\prime}\right)}, \\
& N_{2}(t)=0, \\
& N_{3}(t)=\widetilde{n}_{3} e^{p_{31}\left(-\int_{0}^{\bar{t}_{2}} N_{1}\left(t^{\prime}\right) d t^{\prime}+\int_{0}^{t} N_{1}\left(t^{\prime}\right) d t^{\prime}\right),}
\end{aligned}
$$

for $t \in] 0, T]$. In particular, for $t=\bar{t}_{2}$, one gets

$$
N_{1}\left(\bar{t}_{2}\right)=\widetilde{n}_{1}=n_{1}\left(\bar{t}_{2}\right), \quad N_{2}\left(\bar{t}_{2}\right)=0=n_{2}\left(\bar{t}_{2}\right), \quad N_{3}\left(\bar{t}_{2}\right)=\widetilde{n}_{3}=n_{3}\left(\bar{t}_{2}\right) .
$$

We then conclude, as before, that $n_{2}(0)=N_{2}(0)=0$, which contradicts the fact that $n_{2}(0)>0$. Therefore, there does not exist $\left.\left.\bar{t}_{2} \in\right] 0, T\right]$ such that $n_{2}\left(\bar{t}_{2}\right)=0$, and the continuity of the function $n_{2}(t)$, together with the condition $n_{2}(0)>0$, guarantees that $n_{2}(t)>0$ for all $t \in[0, T]$.

(iii) Finally, we prove that $n_{3}(t)>0$ for all $t \in[0, T]$.

To this end, we rewrite equation (11) in the form

$$
\frac{d}{d t}\left(\ln n_{3}(t)\right)=p_{31} n_{1}(t), \quad t \in[0, T] .
$$

Since $n_{1}(t)$ is positive, we conclude that the function $\ln n_{3}(t)$ is monotonically increasing on $[0, T]$ and the same happens with the function $n_{3}(t)$. Therefore, $n_{3}(t)>0$ for all $t \in[0, T]$. The proof of Lemma 4.1 is then complete.

We will now proceed to analyse the existence and uniqueness of solution to the Cauchy problem (9)-(11), (12), as well as study the asymptotic behaviour of the solution to this system. As we will see, the results crucially depend on the value of the ratio $p_{21} / p_{31}$. See the discussion in paper [4] for a similar problem.

In particular, for $p_{21} / p_{31}<1$, we prove existence and uniqueness of a global solution to the Cauchy problem (9)-(11), (12) and study the asymptotic behaviour, in the following theorem.

Theorem 4.1. Let us assume that the proliferative rates $p_{21}$ and $p_{31}$ are such that $p_{21}<p_{31}$. Then the Cauchy problem (9)-(11) and (12) has a unique solution $\underline{n}(t)=\left(n_{1}(t), n_{2}(t), n_{3}(t)\right)$ defined on all $\mathbb{R}_{+}$. Moreover, this solution satisfies the conditions

$$
\begin{aligned}
\lim _{t \rightarrow+\infty} n_{1}(t) & =0, \\
\lim _{t \rightarrow+\infty} n_{2}(t) & =0, \\
\lim _{t \rightarrow+\infty} n_{3}(t) & =\alpha<+\infty,
\end{aligned}
$$

whatever are the corresponding initial data (12).

Proof.

(i) Firstly, we start by proving that the Cauchy problem (9)-(11), (12) has a unique global (in time) solution. 
Let $J \subset \mathbb{R}_{+}$be the maximal interval on which the solution of problem (9)-(11) and (12) can be defined. By Lemma 4.1, we have that functions $n_{1}(t), n_{2}(t), n_{3}(t)$ are positive for all $t \in J$.

We now put $p_{21}=\lambda p_{31}$, with $0<\lambda<1$, and rewrite equations (10) and (11) in the form

$$
\begin{aligned}
\frac{d}{d t}\left(\ln n_{2}(t)\right) & =\lambda p_{31} n_{1}(t)-d_{23} n_{3}(t), \\
\frac{d}{d t}\left(\ln n_{3}(t)\right) & =p_{31} n_{1}(t) .
\end{aligned}
$$

Moreover, we use equation (11) to obtain

$$
n_{1}(t)=\frac{1}{p_{31}} \frac{\dot{n}_{3}(t)}{n_{3}(t)}
$$

and equation (10) to obtain

$$
\frac{\dot{n}_{2}(t)}{n_{2}(t)}=\lambda p_{31} n_{1}(t)-d_{23} n_{3}(t) .
$$

By combining equations (18) and (19), we can write

$$
\frac{\dot{n}_{2}(t)}{n_{2}(t)} \leq \lambda \frac{\dot{n}_{3}(t)}{n_{3}(t)}
$$

that is

$$
\frac{d}{d t}\left(\ln n_{2}(t)\right) \leq \lambda \frac{d}{d t}\left(\ln n_{3}(t)\right)
$$

and integrating between 0 and $t$, we get

$$
n_{2}(t) \leq C_{1} n_{3}^{\lambda}(t), \quad \text { with } \quad C_{1}=\frac{n_{2}(0)}{n_{3}^{\lambda}(0)} .
$$

Now, from equation (9), using inequality (20), we obtain

$$
\dot{n}_{1} \leq\left(p_{12} C_{1} n_{3}^{\lambda-1}-d_{13}\right) n_{1} n_{3} .
$$

Consequently, if $p_{12} C n_{3}^{\lambda-1}-d_{13} \leq 0$, from inequality (21) we have that $n_{1}(t)$ is a nonincreasing function and therefore $n_{1}(t)$ is bounded, since $0<n_{1}(t) \leq n_{1}(0)$. On the other hand, if $p_{12} C n_{3}^{\lambda-1}-d_{13}>0$, then we use equation (11) to obtain $n_{1}(t) n_{3}(t)=\dot{n}_{3}(t) / p_{31}$ and inequality (21) implies

$$
\dot{n}_{1} \leq\left(\frac{p_{12}}{p_{31}} C_{1} n_{3}^{\lambda-1}-\frac{d_{13}}{p_{31}}\right) \dot{n}_{3}
$$

Integrating between 0 and $t$, we get

$$
n_{1}(t)-n_{1}(0) \leq \frac{C_{1}}{\lambda} \frac{p_{12}}{p_{31}}\left(n_{3}^{\lambda}(t)-n_{3}^{\lambda}(0)\right)-\frac{d_{13}}{p_{31}}\left(n_{3}(t)-n_{3}(0)\right) .
$$

Let us consider now the function $f$ defined on $\mathbb{R}^{+}$by

$$
f(x)=\frac{C_{1}}{\lambda} \frac{p_{12}}{p_{31}} x^{\lambda}-\frac{d_{13}}{p_{31}} x, \quad x \in \mathbb{R}_{0}^{+},
$$


whose first and second order derivatives are, respectively, given by

$$
f^{\prime}(x)=C_{1} \frac{p_{12}}{p_{31}} x^{\lambda-1}-\frac{d_{13}}{p_{31}}, \quad f^{\prime \prime}(x)=C_{1}(\lambda-1) \frac{p_{12}}{p_{31}} x^{\lambda-2}, \quad x \in \mathbb{R}_{0}^{+} .
$$

It is easy to verify that $f$ is bounded from above and therefore we have

$$
f(x) \leq f(\bar{x}), \quad x \geq 0,
$$

where

$$
\bar{x}=\left[\frac{1}{C_{1}} \frac{d_{13}}{p_{12}}\right]^{1 /(\lambda-1)} .
$$

Consequently, by conditions (22), we conclude that

$$
n_{1}(t)-n_{1}(0) \leq f(\bar{x})-f\left(n_{3}(0)\right)<+\infty, \quad \text { for } \quad t \in J .
$$

Inequality (24), together with equations (16) and (17), implies that $J=\mathbb{R}^{+}$, and this proves that our Cauchy problem has a unique solution in $\mathbb{R}^{+}$.

(ii) We now proceed to study the asymptotic behaviour of the solution, by proving conditions (13), (14) and (15). Equations (18) and (19), together with the fact that $n_{3}(t)$ is a monotonous increasing function, give

$$
\frac{\dot{n}_{2}(t)}{n_{2}(t)} \leq \lambda \frac{\dot{n}_{3}(t)}{n_{3}(t)}-C_{2}, \quad \text { for } \quad t \in \mathbb{R}^{+}
$$

Integrating between 0 and $t$, we obtain

$$
n_{2}(t) \leq \frac{n_{2}(0)}{n_{3}^{\lambda}(0)} n_{3}^{\lambda}(t) e^{-C_{2} t}, \quad \text { for } \quad t \in \mathbb{R}^{+},
$$

and this implies that

$$
n_{2}(t) \leq C_{3} n_{3}(t) e^{-C_{2} t}, \quad \text { for } \quad t \in \mathbb{R}^{+},
$$

where $C_{3}=\frac{n_{2}(0)}{n_{3}^{2-\lambda}(0)}$, since $n_{3}(t)$ is monotonously increasing.

Now, by equation (9) together with inequality (25), we have that

$$
\frac{\dot{n}_{1}(t)}{n_{1}(t)} \leq p_{12} C_{3} n_{3}(t) e^{-C_{2} t}-d_{13} n_{3}(t), \quad \text { for } \quad t \in \mathbb{R}^{+} .
$$

Introducing a suitable constant $\beta$ such that $0<\beta<d_{13}$, we arrive at the following inequality

$$
\begin{aligned}
\frac{d}{d t}\left(\ln n_{1}(t)\right) & \leq\left(p_{12} C_{3} e^{-C_{2} t}-d_{13}+\beta\right) n_{3}(t)-\beta n_{3}(t) \\
& \leq\left(p_{12} C_{3} e^{-C_{2} t}-d_{13}+\beta\right) n_{3}(t)-C_{4},
\end{aligned}
$$

where $C_{4}=\beta n_{3}(0)$, since $n_{3}(t)$ is monotonously increasing.

Now, for each $\beta$, we choose $t$ in such a way that $p_{12} C_{3} e^{-C_{2} t}-d_{13}+\beta=0$, and we obtain

$$
t \geq t_{0}, \quad \text { with } \quad t_{0}=\max \{0, \bar{t}\} \quad \text { and } \quad \bar{t}=\frac{1}{C_{2}} \ln \left(\frac{C_{3} p_{12}}{d_{13}-\beta}\right) .
$$


Then, by (26), we arrive at the following inequality

$$
n_{1}(t) \leq n_{1}\left(t_{0}\right) e^{-C_{4}\left(t-t_{0}\right)}, \quad \text { for } \quad t \geq t_{0} .
$$

which proves statement (13) on the asymptotic behaviour of $n_{1}(t)$, since $t_{0}$ is a well defined positive constant and $0<n_{1}\left(t_{0}\right)<+\infty$.

In order to study the asymptotic behaviour of $n_{3}(t)$, we consider equation (11),

$$
\frac{\dot{n}_{3}(t)}{n_{3}(t)}=p_{31} n_{1}(t)
$$

and integrating from 0 and $t$, we get

$$
n_{3}(t)=n_{3}(0) e^{p_{31} \int_{0}^{t} n_{1}\left(t^{\prime}\right) d t^{\prime}},
$$

so that when taking the limit we have

$$
\lim _{t \rightarrow+\infty} n_{3}(t)=n_{3}(0) e^{p_{31} \int_{0}^{\infty} n_{1}\left(t^{\prime}\right) d t^{\prime}}
$$

with

$$
\begin{aligned}
\int_{0}^{+\infty} n_{1}\left(t^{\prime}\right) d t^{\prime} & =\int_{0}^{t_{0}} n_{1}\left(t^{\prime}\right) d t^{\prime}+\int_{t_{0}}^{+\infty} n_{1}\left(t^{\prime}\right) d t^{\prime} \\
& \leq C_{5}+n_{1}(0) \int_{t_{0}}^{+\infty} e^{-C_{4} t^{\prime}} d t^{\prime}=C_{5}+\frac{e^{-C_{4} t_{0}}}{C_{4}} n_{1}(0),
\end{aligned}
$$

where we have used (28) and $t_{0}$ has been defined in (27). Considering (29) and (30), we prove (15) on the asymptotic behaviour of $n_{3}(t)$.

Finally, we analyse the asymptotic behaviour of $n_{2}(t)$. We consider equation (10),

$$
\frac{\dot{n}_{2}(t)}{n_{2}(t)}=p_{21} n_{1}(t)-d_{23} n_{3}(t)
$$

and integrating once more between 0 and $t$, we obtain

$$
n_{2}(t)=n_{2}(0) e^{\int_{0}^{t} p_{21} n_{1}\left(t^{\prime}\right) d t^{\prime}} e^{-\int_{0}^{t} d_{23} n_{3}\left(t^{\prime}\right) d t^{\prime}} \leq n_{2}(0) e^{\int_{0}^{t} p_{21} n_{1}\left(t^{\prime}\right) d t^{\prime}} e^{-\int_{0}^{t} d_{23} n_{3}(0) d t^{\prime}},
$$

since $n_{3}(t)$ is monotonously increasing. We can easily conclude that $\int_{0}^{t} p_{21} n_{1}\left(t^{\prime}\right) d t^{\prime}$ tends to a constant, due to $(30)$, and that $\lim _{t \rightarrow+\infty}\left(-\int_{0}^{t} d_{23} n_{3}(0) d t^{\prime}\right)=-\infty$. Therefore, by taking the limit when $t \rightarrow+\infty$ in the inequality (31), we obtain condition (14) on the asymptotic behaviour of $n_{2}(t)$.

In Theorem 4.1 we prove that the solution of the macroscopic system (9)-(11) is bounded, this in turn implies that the solution to our kinetic system (5)-(7) is also bounded. The boundedness of the solution to the kinetic system is a very important property when solving the system numerically. In the section that follows we perform some numerical simulations based on the kinetic system (5)-(7) in order to study its sensitivity to certain biologically relevant parameters. 


\section{Quantitative analysis and numerical simulations}

In this section, we use the kinetic equations (5)-(7) to perform numerical simulations with the aim of illustrating the sensitivity of the model to certain parameters. In particular, we investigate how the results reproduced by the model are affected when we vary the proliferative rate $p_{31}$ of the ISCs after interacting with SAPCs, the destructive rates $d_{13}$ of SAPCs and $d_{23}$ of SRTCs due to their interaction with ISCs and the value of $w^{*}$ characterizing the capacity of SAPCs to activate SRTCs.

The kinetic system (5)-(7) is solved numerically first by discretizing the equations in the activation state variable (see, e.g. [15]) and then using a quadrature rule to perform the numerical integration of the interaction terms appearing in the equations.

\subsection{The approximation method}

Here we give a brief introduction of the approximation method used to solve equations (5)(7). First, we choose a uniform discrete grid for the activation state variable $u \in[0,1]$ and introduce the set $U$ of $m+1(m \in \mathbb{N})$ equidistant grid points $u_{k} \in[0,1], k=0, \ldots, m$, defined by

$$
u_{k}=k \Delta u
$$

where $\Delta u=1 / m$ is the step size. We assume that the tolerance parameter $w *$, introduced in equation (4), coincides with the grid-point on the $\ell$-position in $U$, that is $w^{*}=u_{\ell}$. The grid points $u_{k}$ are used for the approximation of the distribution function $f_{i}(t, u)$ and we introduce the notation

$$
f_{i}^{k}(t)=f_{i}\left(t, u_{k}\right),
$$

where $i$ stands for the population $p_{i}$ and $k$ indicates the localization of the activation state variable $u \in[0,1]$, with $i=1,2,3$ and $k=0,1, \ldots, m$.

The grid points $u_{k}$ are also used for the approximation of the integral collision terms in equations (5)-(7), using the trapezoidal rule. More specifically, we consider an integral approximation of type

$$
\int_{u_{\alpha}}^{u_{\beta}} g(t, v) d v \approx \mathcal{Q}_{\alpha}^{\beta}[g(t, v)], \quad 0 \leq \alpha<\beta \leq m,
$$

with

$$
\mathcal{Q}_{\alpha}^{\beta}[g(t, v)]=\frac{g\left(t, v_{\alpha}\right)+g\left(t, v_{\beta}\right)}{2} \Delta v+\sum_{s=\alpha+1}^{\beta-1} g\left(t, v_{s}\right) \Delta v, \quad 0 \leq \alpha<\beta \leq m,
$$

to obtain 


$$
\begin{array}{ll}
\int_{0}^{1} f_{j}(t, v) d v \approx \mathcal{Q}_{0}^{m}\left[f_{j}(t, v)\right], & \int_{0}^{u_{k}} f_{j}(t, v) d v \approx \mathcal{Q}_{0}^{k}\left[f_{j}(t, v)\right], \quad j=1,2, \\
\int_{u_{k}}^{1} f_{j}(t, v) d v \approx \mathcal{Q}_{k}^{m}\left[f_{j}(t, v)\right], & \int_{0}^{1} v f_{j}(t, v) d v \approx \mathcal{Q}_{0}^{m}\left[v f_{j}(t, v)\right], \quad j=1,2, \\
\int_{u_{k}}^{1} v f_{j}(t, v) d v & \approx \mathcal{Q}_{k}^{m}\left[v f_{j}(t, v)\right], \quad \int_{0}^{u_{k}} v f_{j}(t, v) d v \approx \mathcal{Q}_{0}^{k}\left[v f_{j}(t, v)\right], \quad j=1,2, \\
\int_{w *}^{1} f_{1}(t, v) d v & \approx \mathcal{Q}_{\ell}^{m}\left[f_{1}(t, v)\right] .
\end{array}
$$

Therefore, the discretization of the activation state variable $u$, combined with approximations (35), allows us to transform system (5)-(7) into a suitable system of $2(m+1)+1$ ordinary differential equations, given by

$$
\begin{aligned}
\frac{d f_{1}^{k}}{d t}(t)= & 2 c_{13} f_{3}(t)\left(\mathcal{Q}_{k}^{m}\left[v f_{1}(t, v)\right]-u_{k} \mathcal{Q}_{k}^{m}\left[f_{1}(t, v)\right]\right)-c_{13} u_{k}^{2} f_{1}^{k}(t) f_{3}(t) \\
& +c_{12}\left[2\left(u_{k} \mathcal{Q}_{0}^{k}\left[f_{1}(t, v)\right]-\mathcal{Q}_{0}^{k}\left[v f_{1}(t, v)\right]\right)-\left(u_{k}-1\right)^{2} f_{1}^{k}(t)\right] \mathcal{Q}_{0}^{m}\left[f_{2}(t, v)\right] \\
& +p_{12} f_{1}^{k}(t) \mathcal{Q}_{0}^{m}\left[f_{2}(t, v)\right]-d_{13} f_{1}^{k}(t) f_{3}(t), \quad k=0, \ldots, m, \\
\frac{d f_{2}^{k}}{d t}(t)= & 2 c_{23} f_{3}(t)\left(\mathcal{Q}_{k}^{m}\left[v f_{2}(t, v)\right]-u_{k} \mathcal{Q}_{k}^{m}\left[f_{2}(t, v)\right]\right)-c_{23} u_{k}^{2} f_{2}^{k}(t) f_{3}(t) \\
& +c_{21}\left[2\left(u_{k} \mathcal{Q}_{0}^{k}\left[f_{2}(t, v)\right]-\mathcal{Q}_{0}^{k}\left[v f_{2}(t, v)\right]\right) \mathcal{Q}_{\ell}^{m}\left[f_{1}(t, v)\right]-\left(u_{k}-1\right)^{2} f_{2}^{k}(t) \mathcal{Q}_{0}^{m}\left[f_{1}(t, v)\right]\right] \\
& +p_{21} f_{2}^{k}(t) \mathcal{Q}_{0}^{m}\left[f_{1}(t, v)\right]-d_{23} f_{2}^{k}(t) f_{3}(t), \quad k=0, \ldots, m, \\
\frac{d f_{3}}{d t}(t)= & p_{31} f_{3}(t) \mathcal{Q}_{0}^{m}\left[f_{1}(t, v)\right] .
\end{aligned}
$$

System (36)-(38) constitutes the numerical scheme for treating the full system (5)-(7).

\subsection{Results and biological interpretation}

We solve numerically the ODE system (36)-(38), using the standard Maple dsolve command with the numeric option.

As initial data, we assume small amounts of SAPCs, SRTCs and ISCs, namely we take $f_{i}(0), i=1,2,3$, to be of the order $10^{-2}$.

Moreover, in all simulations performed here, we fix the values for those parameters that are not investigated, specifically

$$
c_{13}=c_{23}=0.01, \quad c_{12}=5, \quad c_{21}=10, \quad p_{21}=19, \quad p_{12}=1 .
$$

In the first simulation, we consider the values

$$
p_{31}=20, \quad d_{13}=0.35, \quad d_{23}=0.025, \quad w^{\star}=1 / 30,
$$


and the results reproduced by the model are shown in Figure 1. We can see that the ISC population is unable to regulate the autoimmune reaction, either because of insufficient proliferation of ISCs or insufficient destruction of SAPCs and/or SRTCs. The consequence is mass proliferation of SRTCs with mass production of cytokines by these that lead to a full autoimmune cascade and therefore trending to illness.

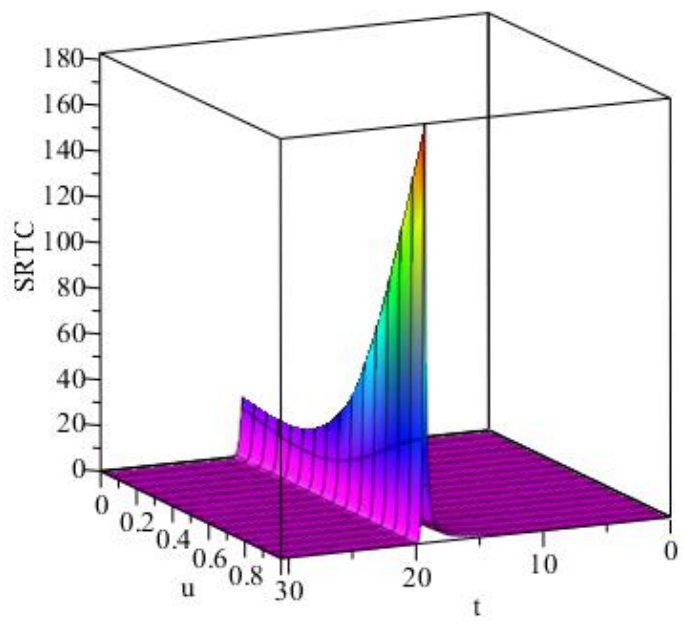

Figure 1: Trending to illness. Evolution of SRTCs densities in the case where the proliferation parameter $p_{31}$ and the destruction rates $d_{13}, d_{23}$ are given by $p_{31}=20, d_{13}=0.35, d_{23}=$ 0.025 , and $w^{\star}=1 / 30$.

In order to control the autoimmune reaction described in Figure 1, we will vary in turn the values of $p_{31}, d_{13}, d_{23}, w^{\star}$ considered in (39). Hence, we will analyse the sensitivity of the model to each of these parameters.

We start by analysing the sensitivity of the model to the proliferation rate $p_{31}$. In order to do so we perform another simulation by changing the value of the proliferation parameter $p_{31}$ given in (39) and considered in Figure 1. Specifically we take

$$
p_{31}=22, \quad d_{13}=0.35, \quad d_{23}=0.025, \quad w^{\star}=1 / 30 .
$$

The results reproduced by the model in this simulation are presented in Figure 2. We can see that, for the values (40), the number of ISCs produced by the biological system are enough to abort the autoimmune reaction in an efficient manner. Hence, illustrating the affect of the proliferation rate $p_{31}$ on the model, in particular on the suppression of the autoimmune reaction.

We now analyse the sensitivity of the model to the destruction rate $d_{13}$. We perform another simulation by changing the value of the destruction parameter $d_{13}$ given in (39) and 


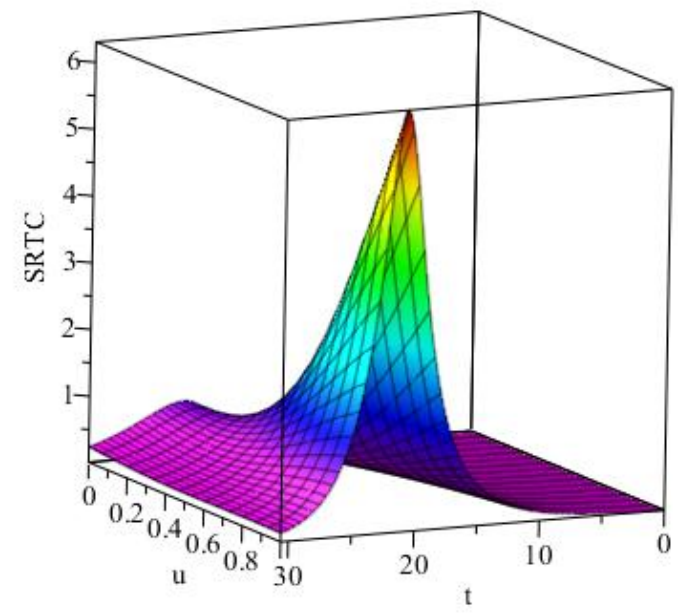

Figure 2: Immunosupression. Evolution of SRTCs densities in the case where the proliferation parameter $p_{31}$ and the destruction rates $d_{13}, d_{23}$ are given by $p_{31}=22, d_{13}=0.35, d_{23}=$ 0.025 , and $w^{\star}=1 / 30$.

considered in Figure 1. We now assume

$$
p_{31}=20, \quad d_{13}=0.6, \quad d_{23}=0.025, \quad w^{\star}=1 / 30 .
$$

The results obtained in this simulation are presented in Figure 3 and show that for the values (41) the number of SAPCs destroyed as a consequence of their interaction with ISCs will ultimately control the proliferation of SRTCs and therefore avoid illness.

Next, in order to analyse the effect of the destruction rate $d_{23}$ on our model, we change the value of the destruction parameter given in (39) and considered in the simulation portrayed in Figure1. More specifically, we take

$$
p_{31}=20, \quad d_{13}=0.35, \quad d_{23}=0.07, \quad w^{\star}=1 / 30 .
$$

The results obtained in this simulation are illustrated in Figure 4. They show that for the values (42), the number of SRTCs destroyed as a consequence of their interaction with ISCs will ultimately avoid a full blown autoimmune reaction.

Finally, if we vary the value of the tolerance parameter $w^{*}$ used in the simulation presented in Figure 1, so that it has a value nearer to one, namely

$$
p_{31}=20, \quad d_{13}=0.35, \quad d_{23}=0.025, \quad w^{\star}=5 / 6,
$$

a significant lowering effect on the number of SRTCs with high activity is observed confirming that the higher the value of $w^{\star}$ the less efficient are SAPCs in increasing the activity of SRTCs, resulting in an increase in the tolerance of SRTCs to self-antigens. This effect is illustrated in Figure 5. 


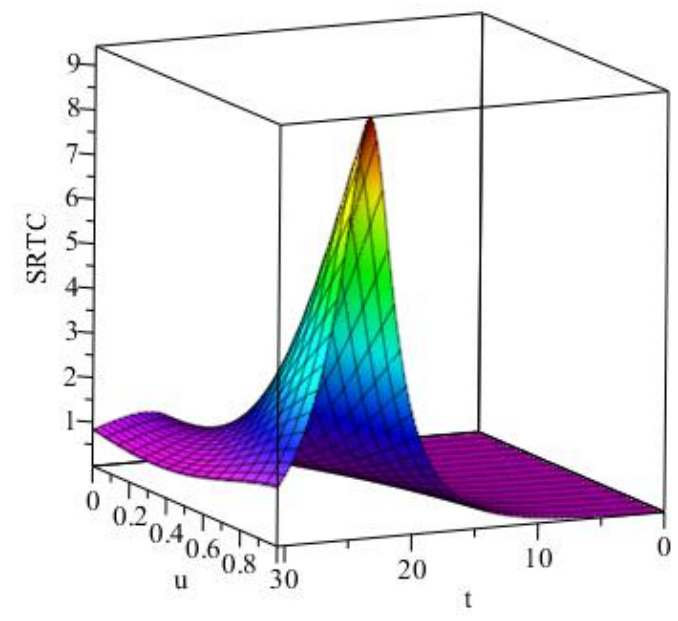

Figure 3: Immunosupression. Evolution of SRTCs densities in the case where the proliferation parameter $p_{31}$ and the destruction rates $d_{13}, d_{23}$ are given by $p_{31}=20, d_{13}=0.6, d_{23}=0.025$, and $w^{\star}=1 / 30$.

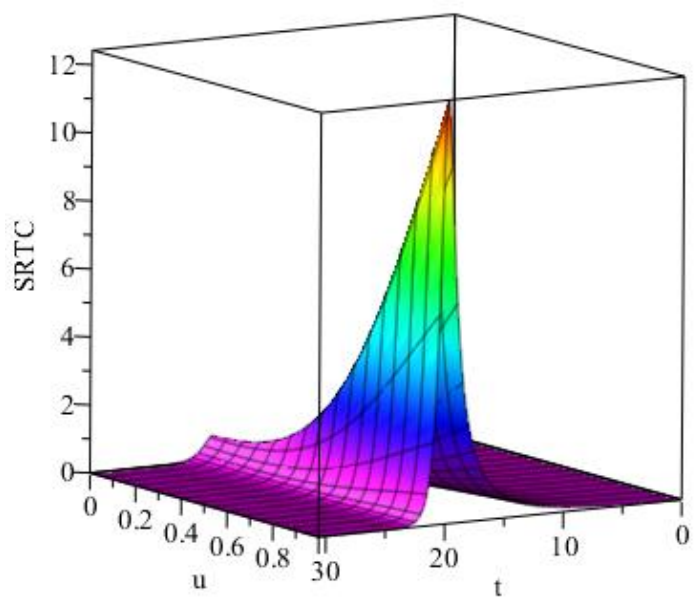

Figure 4: Immunosupression. Evolution of SRTCs densities in the case where the proliferation parameter $p_{31}$ and the destruction rates $d_{13}, d_{23}$ are given by $p_{31}=20, d_{13}=0.35, d_{23}=0.07$, and $w^{\star}=1 / 30$.

\section{$6 \quad$ Summary and perspectives}

In this work we have constructed a new mathematical model, based on the Boltzman-like kinetic theory, describing an autoimmune response against self-antigens. In doing so, we have 


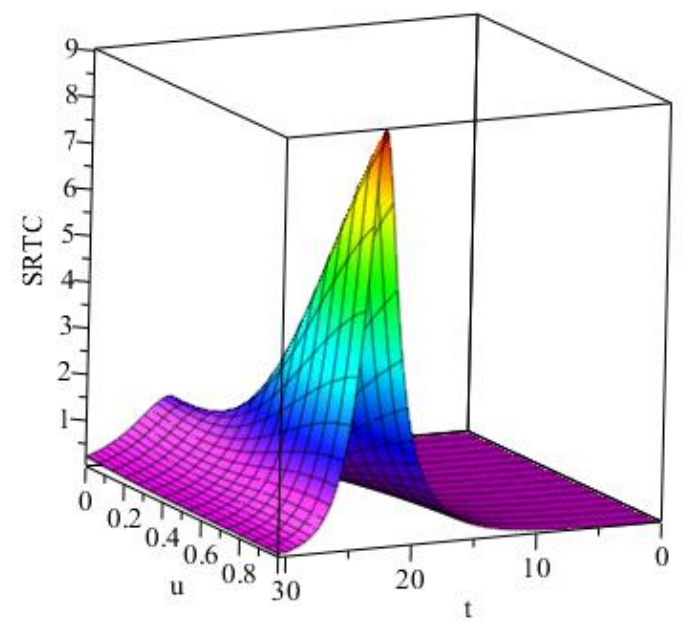

Figure 5: Immunotolerance. Evolution of SRTCs densities in the case where the proliferation parameter $p_{31}$ and the destruction rates $d_{13}, d_{23}$ are given by $p_{31}=20, d_{13}=0.35, d_{23}=0.025$ and $w^{\star}=5 / 6$.

considered three interacting cell populations, which are believed to be the main players in the autoimmune process, these being SAPCs, SRTCs and ISCs (such as Tregs and NKCs).

For all participating cell populations, we have defined an activity variable based on their biological function.

By describing the binary cell interactions, we were able to derive the kinetic equations that give the evolution of the distribution functions associated to the interacting cell populations. The corresponding kinetic system consists of integro-differential equations for the distribution functions whose integral operators describe the binary interactions among the cell populations.

From the kinetic equations, we have formally derived the macroscopic balance equations for the total number of cells of each population and the corresponding system consists, in our case, of ordinary differential equations.

In our model we have assumed that during proliferative encounters cloned cells inherit the same aggressive state as their mother cell, at a constant proliferation rate, and, additionally, that the destructive encounters occur at a constant destruction rate. Under these assumptions, the boundedness of the solution to our macroscopic system implies the boundedness of the solution to the corresponding kinetic system. In fact, the basic information on the kinetic model is contained in the corresponding macroscopic system.

We have, therefore, presented a qualitative analysis of the macroscopic system under a certain assumption on two of the proliferation parameters of the model. Under this assumption, we have proved the existence and uniqueness of a global solution to the Cauchy problem associated to our macroscopic system. We have also studied the asymptotic behaviour of the solution. 
Some numerical simulations of the kinetic model have been included, in order to study the sensitivity of the model to parameters characterizing proliferation of ISCs, destruction of SAPCs and SRTCs and tolerance of SRTCs to self-antigens. The results reproduced by the model have a biological counterpart consistent with the scientific descriptions reported in the literature. Therefore, in our opinion, the model proposed in this paper results to be a suitable and consistent mathematical tool to mimic the biological trends typical of an autoimmune disease.

Indeed, starting from the present study, we plan, in our future work, to extend our model to describe immunotherapy in the context of autoimmune disease and to include an artificial inlet which represents an external drug therapy. Moreover, using optimal control theory, we plan to establish optimal treatment strategies to decrease the number of SRTCs.

\section{Acknowledgments}

This work is partially supported by the Portuguese FCT Project UID/MAT/00013/2013 of CMAT-UM.

\section{References}

[1] B. Akkaya, Y. Oya, M. Akkaya, J. A. Souz, A. H. Holstein, O. Kamenyeva, J. Kabat, R. Matsumura, D. W. Dorward, D. D. Glass and E. M. Shevach, "Regulatory T cells mediate specific suppression by depleting peptide MHC class II from dendritic cells", Nature Immunology, 20, 218-231 (2019).

[2] L. Arlotti and N. Bellomo. "Population dynamics with stochastic interaction", Transp. Theory Statist. Phys., 24, 431-443 (1995).

[3] L. Arlotti, N. Bellomo and K. Latrach. "From the Jager and Segel model to kinetic population dynamics nonlinear evolution problems and applications", Mathl. Comput. Modelling, 30, 15-40 (1999).

[4] L. Arlotti and M. Lachowicz. "Qualitative analysis of a nonlinear integrodifferential equation", Modeling Tumor-Host Dynamics", Mathl. Comput. Modelling, 23, 11-29 (1996).

[5] N. Bellomo, A. Elaiw, A. M. Althiabi and M. A. Alghamdi, "On the interplay between mathematics and biology: Hallmarks toward a new systems biology", Phys. Life Rev., 12, 44-64 (2015).

[6] N. Bellomo and G. Forni. "Dynamics of tumor interaction with the host immune system", Mathl. Comput. Modelling, 20, 107-122 (1994).

[7] N. Bellomo and L. Preziosi. "Modelling and mathematical problems related to tumor evolution and its interaction with the immune system", Mathl. Comput. Modelling, 32, $413-452$ (2000).

[8] P. Bhargava and P. A. Calabresi "Novel therapies for memory cells in autoimmune diseases" Clinical and Experimental Immunology, 180, 353-360 (2015). 
[9] T. M. Brusco, A. L. Putnam and J. A. Bluestone. "Human regulatory T cells: role in autoimmune disease and therapeutic opportunities", Immunological Reviews, 223, 371-390 (2008).

[10] C. Cercignani. Theory and application of the Boltzmann equation, Scotish Academic Press (1975).

[11] N. A. Danke, D. M. Koelle, C. Yee, S. Beheray and W. W. Kwok, "Autoreactive T cells in healthy individuals", The Journal of Immunology, 172, 5967-5972 (2004).

[12] M. Delitala, U. Dianzani, T. Lorenzi and M. Melensi. "A mathematical model for immune and autoimmune response mediated by T-cells", Computers and Mathematics with Applications, 66, 1010-1023 (2013).

[13] P. Devarajan and Z. Chen, "Autoimmune effector memory T cells: the bad and the good", Immunol Res., 57, 12-22 (2013).

[14] P. Hartman. Ordinary differential equations, SIAM (2002).

[15] C. L. Jorcyk, M. Kolev, K. Tawara and B. Zubik-Kowal. "Experimental versus numerical data for breast cancer progression", Nonlinear Analysis: Real World Applications, 13, 78-84 (2012).

[16] C. Kim and M. A. Williams. "Nature and nurture: T-cell receptor-dependent and T-cell receptor-independent differentiation cues in the selection of the memory T-cell pool", Immunology, 131, 310-317 (2010).

[17] M. Kolev and I. Nikolova. "Dynamical properties of autoimmune disease models: Tolerance, flare-up, dormancy", Journal of Theoretical Biology, 246, 646-659 (2007).

[18] M. Kolev and I. Nikolova. Mathematica Applicanda, "A mathematical model of some viral-induced autoimmune diseases. 46, 97-108 (2018).

[19] F. G. Lakkis and M. H. Sayegh. "Memory T cells: a hurdle to immunologic tolerance", J. Am. Soc. Nephrol., 14, 2402-2410 (2003).

[20] R. Liberal, C. R. Grant, M. S. Longhi, G. Mieli-Vergani and D. Vergani. "Regulatory T cells: mechanisms of suppression and impairment in autoimmune liver disease", International Union of Biochemistry and Molecular Biology, 67, 88-97 (2015).

[21] F. Mollica, L. Preziosi, K. R. Rajagopal (Eds.), Modeling of Biological Materials, Series in Modeling and Simulation in Science, Engineering and Technology, Birkhäuser (2007).

[22] B. Perthame, Parabolic Equations in Biology, Lecture Notes on Mathematical Modelling in the Life Sciences, Springer (2015).

[23] A. Poggi and M. R. Zocchi. "NK cell autoreactivity and autoimmune diseases", Frontiers in Immunology, 5, 1-15 (2014).

[24] A. Sharabi, M. G. Tsokos, Y. Ding, T. R. Malek, D. Klatzmann and G. C. Tsokos "Regulatory T cells in the treatment of disease" Nature Reviews Drug Discovery, 17, 823-844 (2018). 
[25] F. Shi and L. V. Kaer "Reciprocal regulation between natural killer cells and autoreactive T cells" Nature Reviews Immunology, 6, 751-760 (2006).

[26] H. R. Thieme, Mathematics in population biology, Princeton series in theoretical and computational biology. Princeton University Press, Princeton (2003).

[27] Z. Tian, M. E. Gershwin and C. Zhang. "Regulatory NK cells in autoimmune disease", Journal of Autoimmunity, 39, 206-215 (2012).

[28] W. Zhang, L. M. Wahl and P. Yu, "Modelling and analysis of recurrent autoimmune disease" Siam J. Appl. Math., 74, 1998-2025 (2014).

[29] A. L. Zozulya and H. Wiendl, "The role of regulatory T cells in multiple sclerosis", Nature Clinical Practice Neurology, 4, pages 384-398 (2008). 